\title{
EFEITO DE PARÂMETROS DE EXTRUSÃO NA COR E PROPRIEDADES DE PASTA DA FARINHA DE MANDIOQUINHA-SALSA (Arracacia xanthorrhiza)
}

\author{
Effect of extrusion parameters on color and pasting properties \\ of peruvian carrot flour (Arracacia xanthorrhiza)
}

\author{
Bruna Menegassi ${ }^{1}$, Magali Leonel ${ }^{2}$, Martha Maria Mischan ${ }^{3}$, Sheila Zambello de Pinho
}

\begin{abstract}
RESUMO
Processou-se neste trabalho a farinha de mandioquinha-salsa (Arracacia xanthorrhiza Bancr.) em uma linha de extrusão (mono rosca) variando as condições operacionais: umidade da farinha (11-19\%), temperatura de extrusão (86-154 $\left.{ }^{\circ} \mathrm{C}\right)$ e taxa de rotação da rosca (136-272rpm). Os parâmetros de cor analisados foram luminosidade ( $\left.\mathrm{L}^{*}\right)$ e os componentes de cromaticidade $\mathrm{a}^{*} \mathrm{e} \mathrm{b}^{*}$. Os parâmetros de propriedade de pasta analisados foram viscosidade inicial, pico de viscosidade, quebra de viscosidade, tendência a retrogradação e viscosidade final. Os resultados obtidos mostraram que a umidade da matéria-prima interferiu nos componentes de cor das farinhas com efeito significativo sobre a luminosidade e croma $a^{*}$, e a temperatura interferiu no croma $b^{*}$. Quanto ao efeito dos parâmetros de processo sobre as propriedades de pasta, a umidade interferiu nas viscosidades inicial e final dos produtos, pico e quebra de viscosidade, enquanto a temperatura de extrusão e a rotação da rosca tiveram influência sobre a tendência a retrogradação e viscosidade final dos produtos.
\end{abstract}

Termos para indexação: Amido, viscosidade, temperatura, Arracacia xanthorrhiza.

\section{ABSTRACT}

In this work peruvian carrot flour (Arracacia xanthorrhiza Bancr.) was processed in a single screw extruder at different moisture contents $(11-19 \%)$, extrusion temperature $\left(86-154^{\circ} \mathrm{C}\right)$ and screw speed $(136-272 \mathrm{rpm})$. The parameters $\mathrm{L}^{*}, \mathrm{a}^{*}$ and $\mathrm{b}^{*}$ of color were analyzed in extruded flours. The viscosity related parameters determined include initial viscosity, viscosity peak, breakdown, setback and final viscosity. The results showed effect of feed moisture on flour color ( $\mathrm{L}^{*}$ and $\left.\mathrm{a}^{*}\right)$ and the extrusion temperature influenced $b^{*}$. Moisture content of the feed had effect on initial and final viscosity, viscosity peak and breakdown. Extrusion temperature and screw speed had effect on final viscosity and setback.

Index terms: Starch, viscosity, temperature, Arracacia xanthorrhiza.

(Recebido em 10 de janeiro de 2006 e aprovado em 26 de junho de 2007)

\section{INTRODUÇÃO}

Embora os cereais representem a principal fonte de carboidratos para a maioria da população mundial, os tubérculos e as raízes constituem também uma importante fonte de alimentos energéticos para diversos povos, sobretudo nas regiões tropicais e subtropicais do planeta.

O processamento de tuberosas no Brasil ainda pode ser considerado pequeno, com exceção da mandioca, que com a industrialização, principalmente para a produção de fécula, aumentou significativamente nos últimos anos, proporcionando a entrada de multinacionais no setor. As demais tuberosas são comercializadas como oleráceas, sendo a mandioquinha-salsa (Arracacia xanthorrhiza Bancr.) de grande expressão. Seu valor alimentício é alto, sendo rica em minerais como cálcio, fósforo e ferro, além de constituir boa fonte de vitaminas do Complexo B, principalmente niacina (PEREIRA, 1997).
A mandioquinha-salsa apresenta importância econômica elevada, com volume de comercialização em torno de 90.000 toneladas/ano e valor ao redor de 50 milhões de dólares (CÂMARA \& SANTOS, 2002). Durante a colheita da mandioquinha-salsa são gerados resíduos: a parte aérea, as raízes fora de padrão e a chamada "cabeça" ou coroa, porção tuberosa da qual saem as raízes, em número de quatro a dez. Esses resíduos têm sido utilizados na alimentação animal. A produção de farinha da coroa e das raízes fora de padrão para uso em produtos alimentícios é uma interessante alternativa para a valorização da cultura.

A tecnologia da extrusão, nos últimos tempos, tem se tornado um dos principais processos no desenvolvimento de produtos alimentícios. A extrusão é um processo contínuo, no qual a matéria-prima é forçada através de uma matriz ou molde, em condições de mistura e aquecimento, pressão e fricção que levam à gelatinização

\footnotetext{
${ }^{1}$ Aluna Graduação Nutrição-IB/UNESP - Botucatu, SP.

2Pesquisadora - Centro de Raízes e Amidos Tropicais/UNESP - Cx. P. 237 - 18603-970 - Botucatu, SP - mleonel@fca.unesp.br

${ }^{3}$ Professora Doutora - Departamento de Bioestatística - IB/UNESP - Botucatu, SP.
} 
do amido, a desnaturação de proteínas e à ruptura de pontes de hidrogênio (THAKUR \& SAXENA, 2000).

O grânulo de amido natural tem uma capacidade limitada de absorver água fria. Durante o cozimento por extrusão, o amido que apresenta inicialmente uma forma granular, é progressivamente comprimido e transformado em um material denso, sólido e compacto, desaparecendo sua estrutura cristalina e granular. As principais propriedades funcionais do amido extrusado quando dispersos em água, são a absorção e a solubilidade em água. Ele absorve água rapidamente, formando uma pasta à temperatura ambiente, sem qualquer aquecimento. $\mathrm{O}$ aumento da solubilidade com a gelatinização é a base para a confecção de alimentos amiláceos pré-preparados (CHANG et al., 2001).

No mercado alimentício não há muitos produtos à base de mandioquinha-salsa, ficando restrita ao consumo culinário. Unindo-se a tecnologia da extrusão à importância sócio-econômica do desenvolvimento de produtos de elevado valor agregado a partir de novas matérias-primas e a agregação de valor a resíduos, objetivou-se neste trabalho avaliar o efeito das condições de extrusão sobre a cor e as propriedades viscográficas da farinha de mandioquinha-salsa extrusada.

\section{MATERIAL E MÉTODOS}

Uma quantidade de $300 \mathrm{~kg}$ de mandioquinha-salsa, variedade Senador Amaral colhida com 12 meses do plantio e apresentando $74 \%$ de umidade, foi processada para a obtenção de aproximadamente $70 \mathrm{~kg}$ de farinha. Para padronizar a granulometria, a farinha foi classificada em um agitador de peneiras utilizando-se uma peneira de 20 mesh.

A extrusão foi efetuada em uma linha completa de extrusão IMBRA RX da Imbramaq S/A, com motor de 10HP acoplado a redutor de velocidade, sistema de extrusão através de fricção mecânica, rosca simples de extrusão, sistema de refrigeração hidráulica, para controle de temperatura na camisa de extrusão, velocidade variável e capacidade de produção de $45 \mathrm{~kg} / \mathrm{h}$. Os parâmetros de processamento foram divididos em fixos e variáveis. Os parâmetros fixos foram:

- taxa de compressão da rosca (4,5 mm profundidade e 14 mm de largura): 3:1;

- taxa de alimentação: $200 \mathrm{~g} / \mathrm{min}$;

- abertura da matriz: $3 \mathrm{~mm}$;

- temperatura na $1^{\mathrm{a}}$ zona: $20^{\circ} \mathrm{C}$;

- temperatura na $2^{\mathrm{a}}$ zona: $70^{\circ} \mathrm{C}$.
Os parâmetros variáveis foram: temperatura na terceira zona do extrusor, umidade das farinhas e rotação da rosca.

A cor das farinhas extrusadas foi avaliada em colorímetro Minolta Chroma Meter CR 200b. Os resultados foram expressos em valores $\mathrm{L}^{*}, \mathrm{a}^{*} \mathrm{e} \mathrm{b}^{*}$, onde os valores de $\mathrm{L}^{*}$ (luminosidade ou brilho) variam do preto (0) ao branco (100), os valores do croma $a^{*}$ variam do verde (-60) ao vermelho (+60) e os valores do croma $b^{*}$ variam do azul ao amarelo, ou seja, de -60 a +60 , respectivamente.

Para a avaliação das propriedades de pasta das farinhas de mandioquinha-salsa foi utilizado o Rapid Visco Analyser (RVA), série 4, da Newport Scientific, e considerou-se a concentração de $3,5 \mathrm{~g}$ de farinha/ $25 \mathrm{~mL}$ de água destilada, corrigida para a base de $14 \%$ de umidade (NEWPORT SCIENTIFIC, 1998). Por causa da formação de grumos nas farinhas extrusadas, adicionouse $1 \mathrm{~mL}$ de etanol às amostras antes da diluição para a correção da umidade.

Foi utilizada a programação Extrusion 2 (com álcool) do software Thermocline for Windows, versão 2.2 para as farinhas extrusadas. A viscosidade foi expressa nas unidades do aparelho, ou seja, Rapid Visco Units (RVU).

Os parâmetros avaliados foram: viscosidade inicial (VI), que é o pico de viscosidade entre o tempo $0,2-2$ minutos; pico de viscosidade (PV), que é a viscosidade máxima obtida após o início do aquecimento e antes do início do resfriamento; quebra de viscosidade (QV), que é a diferença entre a viscosidade máxima e mínima durante a manutenção a $95^{\circ} \mathrm{C}$ (hold); tendência à retrogradação (TR), que é a diferença entre a viscosidade final e o menor valor de viscosidade durante a manutenção a $95^{\circ} \mathrm{C}$ (hold); e viscosidade final (VF).

Para analisar o efeito combinado das variáveis independentes nas características tecnológicas dos extrusados utilizou-se o delineamento 'central composto rotacional' para três fatores, segundo Cochran \& Cox (1957), com um total de 15 tratamentos (Tabela 1), a saber: - 8 tratamentos correspondentes ao fatorial $2^{3}$, onde os três fatores são:

$\mathrm{R}(\mathrm{rpm})=$ rotação da rosca, $\mathrm{T}=$ temperatura $\left({ }^{\circ} \mathrm{C}\right), \mathrm{U}=$ umidade (\%), cada qual em dois níveis, codificados como $-1 \mathrm{e}+1$;

- 6 tratamentos com níveis mínimo e máximo de cada fator, codificados como $-\alpha$ e $+\alpha$, respectivamente, sendo $\alpha=2^{3 / 4}=1,682$;

- Um tratamento central repetido 6 vezes, onde os fatores estão todos em um nível médio, codificado como zero. 
Tabela 1 - Parâmetros variáveis do processo de extrusão.

\begin{tabular}{ccccc}
\hline & Níveis & & Fatores ou variáveis independentes & \\
\hline Axiais & Codificados & $\mathrm{R}$ & $\mathrm{T}$ & $\mathrm{U}$ \\
$-\alpha$ & $-1,682$ & 136 & 86 & 11 \\
& -1 & 163 & 100 & 13 \\
& 0 & 204 & 120 & 15 \\
& +1 & 245 & 140 & 17 \\
$+\alpha$ & $+1,682$ & 272 & 154 & 19 \\
\hline
\end{tabular}

R: Rotação da rosca (rpm); T: Temperatura de extrusão $\left({ }^{\circ} \mathrm{C}\right)$; U: Umidade das amostras (\%).

Para a análise estatística dos resultados experimentais utilizou-se o modelo

$$
\mathrm{y}_{\mathrm{k}}=\mathrm{b}_{0}+\sum_{\mathrm{i}=1}^{3} \mathrm{~b}_{\mathrm{i}} \mathrm{x}_{\mathrm{ik}}+\sum_{\mathrm{i}=1}^{3} \sum_{\mathrm{j}=1}^{3} \mathrm{~b}_{\mathrm{ij}} \mathrm{x}_{\mathrm{ik}} \mathrm{x}_{\mathrm{jk}}+\mathrm{e}_{\mathrm{k}}
$$

onde:

$\mathrm{y}_{\mathrm{k}}=$ valor observado da variável dependente no nível $k$, $\mathrm{k}=1, \ldots, 20$;

$\mathrm{x}_{\mathrm{ik}}=\mathrm{i}$-ésima variável independente, no nível $k, \mathrm{i}=1,2,3$;

$\mathrm{b}_{0}=$ parâmetro do modelo, independente de $x$;

$\mathrm{b}_{\mathrm{i}}=$ parâmetros correspondentes aos efeitos lineares de $\mathrm{x}_{\mathrm{i}}$;

$\mathrm{b}_{\mathrm{ij}}=$ parâmetros correspondentes aos efeitos de $2^{\mathrm{a}}$ ordem

$\operatorname{de}_{\mathrm{i}} \mathrm{x}_{\mathrm{j}}, \mathrm{i}=1,2,3, \mathrm{j}=\mathrm{i}, \ldots, 3$;

$\mathrm{e}_{\mathrm{k}}=$ erro experimental associado à $\mathrm{k}$-ésima parcela.

O processamento dos dados e a análise estatística foram realizados com o auxílio do sistema SAS. A significância do modelo foi testada pela análise de variância (ANOVA), sendo adotado o nível de significância alfa de $5 \%$.

\section{RESULTADOS E DISCUSSÃO}

\section{Análise de cor}

A cor adquirida pelos produtos extrusados está relacionada à caramelização e a reação de Maillard, principalmente em materiais que apresentam teores relativamente altos de açúcares totais.

O componente $\mathrm{L}^{*}$ das farinhas extrusadas variou de 65 a 76, indicando redução da luminosidade com a extrusão, quando comparado com a luminosidade da farinha crua que foi 85,8 . Entre os parâmetros que compõem o modelo de regressão apenas a umidade afetou significativamente a luminosidade, ao passo que os parâmetros temperatura e rotação da rosca não apresentaram efeito sobre essa característica. $O$ coeficiente de determinação foi de $65,5 \%$ (Tabela 2).

Nota-se pela Figura 1, o efeito da umidade das amostras sobre o componente $\mathrm{L}^{*}$ de cor das farinhas extrusadas quando a temperatura e a rotação da rosca foram mantidas no ponto central do delineamento $\left(120^{\circ} \mathrm{C}\right.$ e $204 \mathrm{rpm}$ ). Foi observado aumento do valor de $\mathrm{L}^{*}$ com o aumento da umidade até $15 \%$, e a partir daí observou-se uma tendência à diminuição desses valores.

Gutkoski \& El-Dash (1999) estudando produtos extrusados de aveia, com umidade variando de 17 a $24 \%$ e a temperatura de extrusão de $90^{\circ} \mathrm{C}$ a $150{ }^{\circ} \mathrm{C}$, com rotação constante de $100 \mathrm{rpm}$, verificaram que a luminosidade diminuiu linearmente com o aumento da umidade e com o aumento da temperatura de extrusão.

Borba et al. (2005) avaliando o efeito de parâmetros operacionais na extrusão de farinha de batata-doce observaram aumento do componente $\mathrm{L}^{*}$ com o aumento da umidade até $20-21 \%$, com temperatura de $120^{\circ} \mathrm{C}$ e rotação de $210 \mathrm{rpm}$. Os valores observados variaram de 81 a 88 nas farinhas extrusadas e o da farinha crua foi de 94,6 .

A coordenada de cromaticidade $a^{*}$ das farinhas extrusadas apresentou valores que variaram de 4 a 10 , valores superiores ao da farinha crua que foi de 1,72. Considerando que esse componente de cor varia de verde $(-60)$ ao vermelho $(+60)$, pode-se dizer que ocorreu pequena variação para o vermelho, entre as farinhas.

O modelo de regressão adotado para a variável, $\mathrm{a}^{*}$, não foi significativo $(p>0,05)$ nessas condições experimentais. Entre os fatores que compõem o modelo, apenas a umidade interferiu de forma significativa para esse componente de cromaticidade das farinhas extrusadas. O coeficiente de determinação foi de 72,2\% (Tabela 3). 
Tabela 2 - Coeficientes de regressão e ANOVA para o componente L* das farinhas extrusadas.

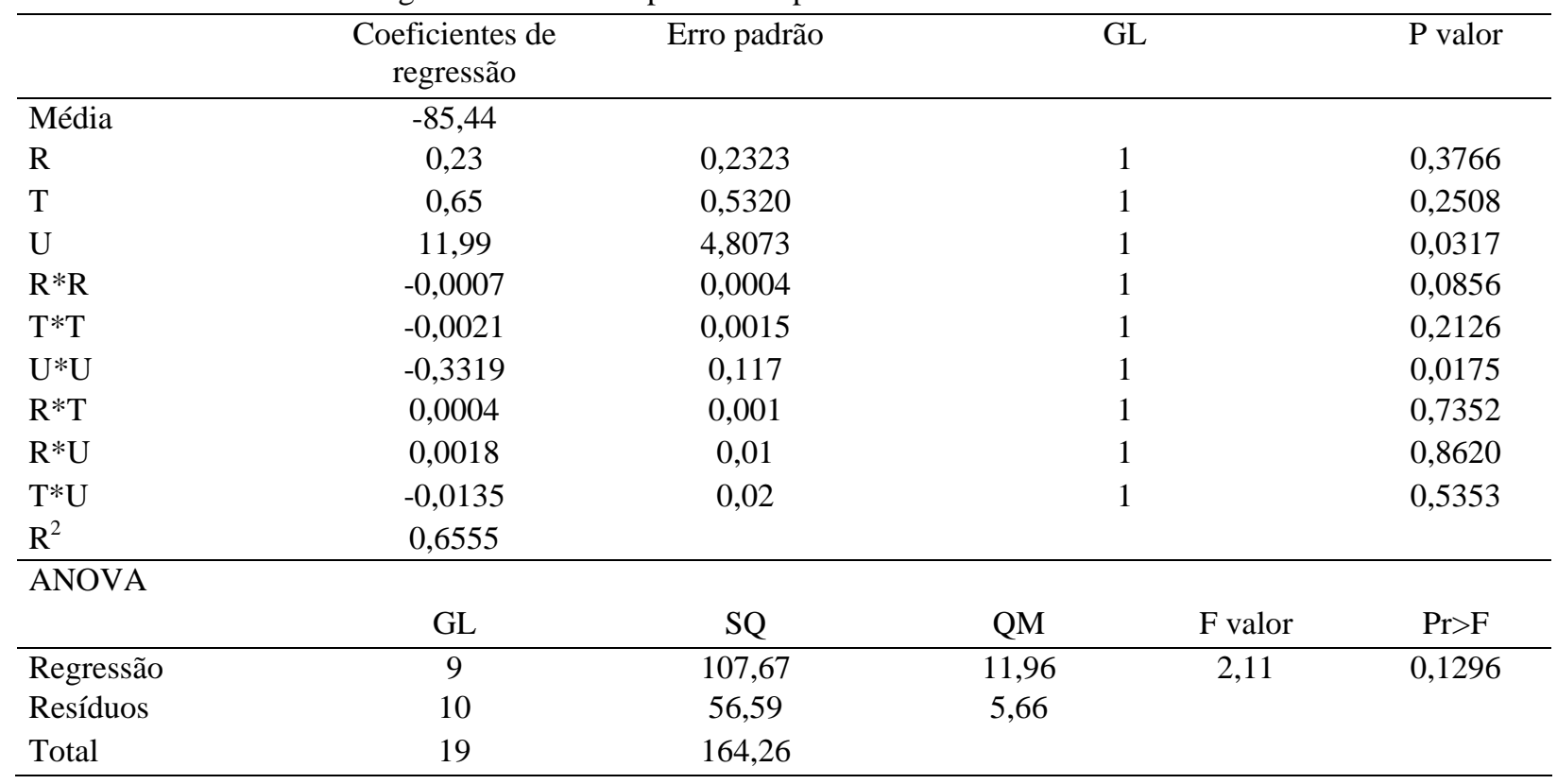

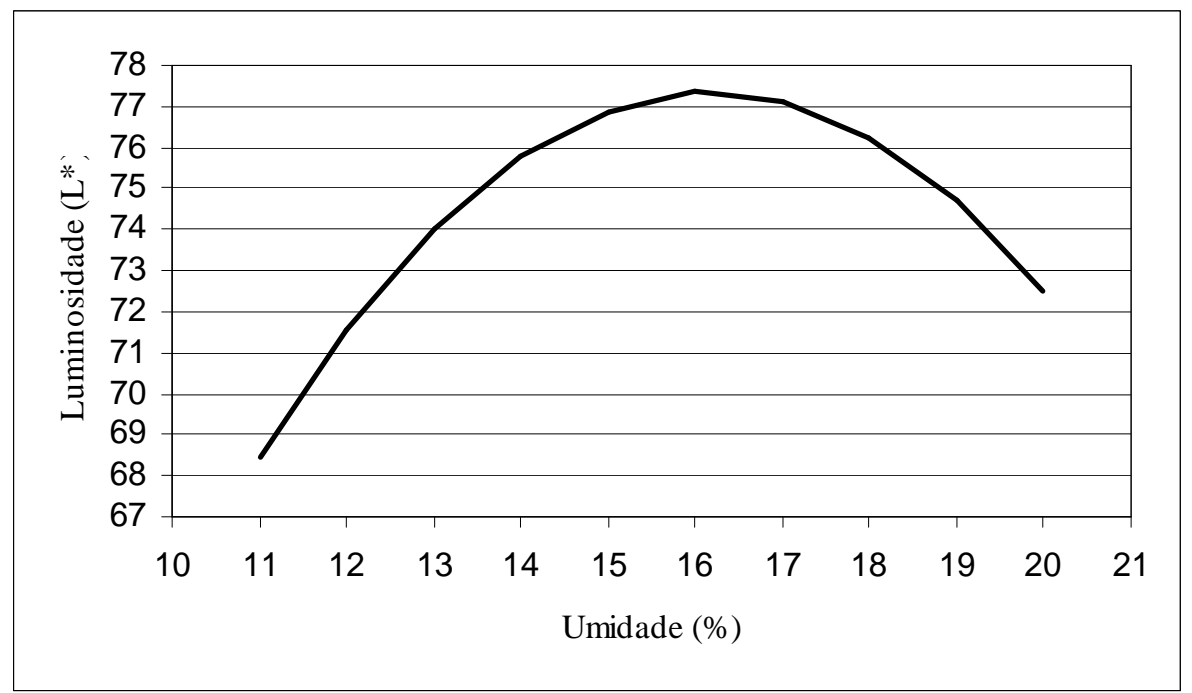

Figura 1 - Efeito do teor de umidade da matéria-prima sobre o componente $\mathrm{L}^{*}$ das farinhas extrusadas, sob condições de temperatura de $120^{\circ} \mathrm{C}$ e rotação da rosca de $204 \mathrm{rpm}$.

Como observa-se na Figura 2, os maiores valores de croma a* foram obtidos nas condições de baixa umidade, onde também foram observados os menores valores de $\mathrm{L}^{*}$, indicando a ocorrência de reações de escurecimento não enzimático.
Já a coordenada de cromaticidade $b^{*}$, que representa o índice de amarelo que vai de azul (-60) até amarelo (+60), variou de 30 a 37 , mostrando forte presença do amarelo em todas as farinhas extrusadas. A farinha de mandioquinha-salsa antes da extrusão apresentava valor de croma b* de 15,75. 
MENEGASSI, B. et al.

Tabela 3 - Coeficientes de regressão e ANOVA para o componente a* das farinhas extrusadas.

\begin{tabular}{|c|c|c|c|c|c|}
\hline & $\begin{array}{l}\text { Coeficientes de } \\
\text { regressão }\end{array}$ & Erro padrão & \multicolumn{2}{|c|}{ GL } & $\mathrm{P}$ valor \\
\hline Média & \multicolumn{4}{|l|}{90,07} & \\
\hline $\mathrm{R}$ & $-0,074$ & \multicolumn{3}{|l|}{0,1363} & 0,5995 \\
\hline $\mathrm{T}$ & $-0,38$ & \multicolumn{3}{|l|}{0,2874} & 0,2149 \\
\hline $\mathrm{U}$ & $-6,61$ & \multicolumn{3}{|l|}{2,60} & 0,0291 \\
\hline $\mathrm{R} * \mathrm{R}$ & 0,0003 & \multicolumn{3}{|l|}{0,0002} & 0,1751 \\
\hline $\mathrm{T}^{*} \mathrm{~T}$ & 0,001 & \multicolumn{3}{|l|}{0,0008} & 0,2522 \\
\hline $\mathrm{U} * \mathrm{U}$ & 0,178 & \multicolumn{3}{|l|}{0,063} & 0,0181 \\
\hline $\mathrm{R} * \mathrm{~T}$ & $-0,0001$ & \multicolumn{3}{|l|}{0,0005} & 0,8574 \\
\hline $\mathrm{R} * \mathrm{U}$ & $-0,002$ & \multicolumn{3}{|l|}{0,005} & 0,6672 \\
\hline $\mathrm{T}^{*} \mathrm{U}$ & 0,0096 & \multicolumn{3}{|l|}{0,011} & 0,4181 \\
\hline $\mathrm{R}^{2}$ & 0,7218 & & & & \\
\hline \multicolumn{6}{|l|}{ ANOVA } \\
\hline & GL & SQ & QM & F valor & $\operatorname{Pr}>F$ \\
\hline Regressão & 9 & 42,85 & 4,76 & \multirow[t]{3}{*}{2,88} & \multirow[t]{3}{*}{0,0572} \\
\hline Resíduos & 10 & 16,51 & 1,65 & & \\
\hline Total & 19 & 59,36 & & & \\
\hline
\end{tabular}

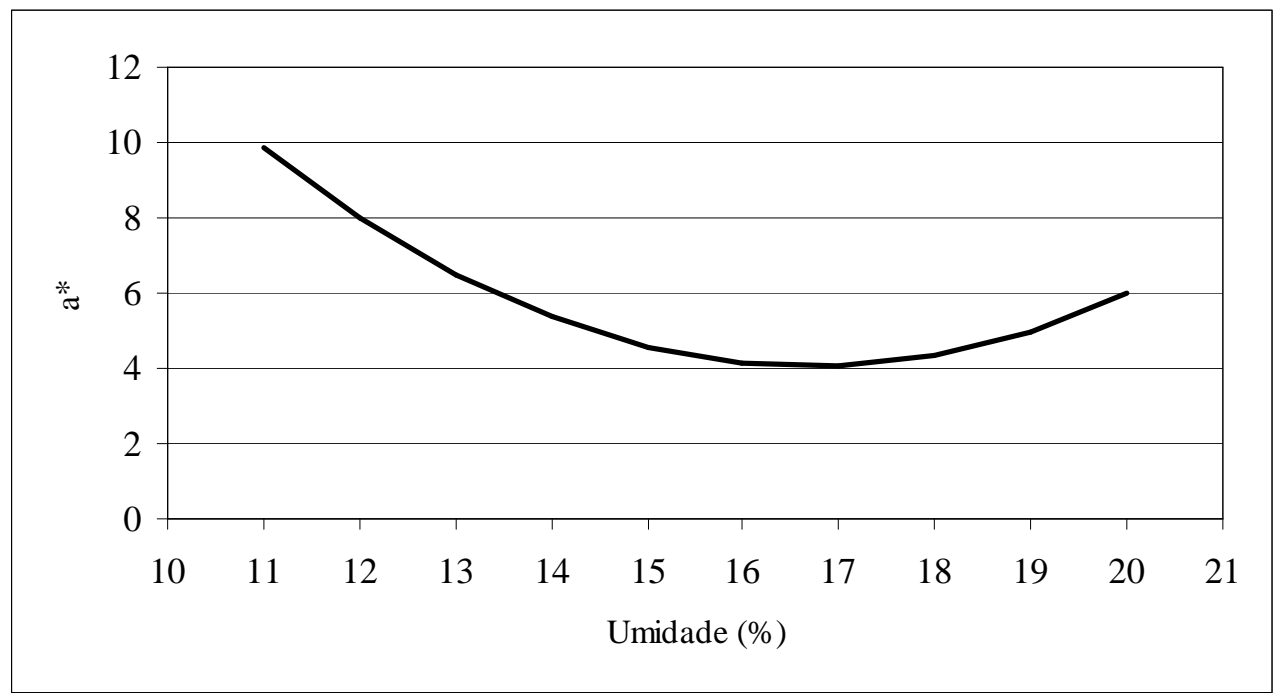

Figura 2 - Efeito do teor de umidade da matéria-prima sobre o componente a* das farinhas extrusadas, sob condições de temperatura de $120^{\circ} \mathrm{C}$ e rotação da rosca de $204 \mathrm{rpm}$.

Os coeficientes de regressão obtidos indicaram que a temperatura interferiu de forma significativa sobre o componente $b^{*}$ das farinhas extrusadas, mostrando menores valores do croma $b^{*}$ na condição intermediária de temperatura (Tabela 4 e Figura 3).
Segundo Badrie \& Mellowes (1991) a elevação da temperatura aumenta a intensidade da cor e altos níveis de umidade resultam em produtos mais claros, pois o aumento da umidade reduz o tempo de residência, proporcionando menor ocorrência de caramelização e reação de Maillard. 
Tabela 4 - Coeficientes de regressão e ANOVA para o componente $b^{*}$ das farinhas extrusadas.

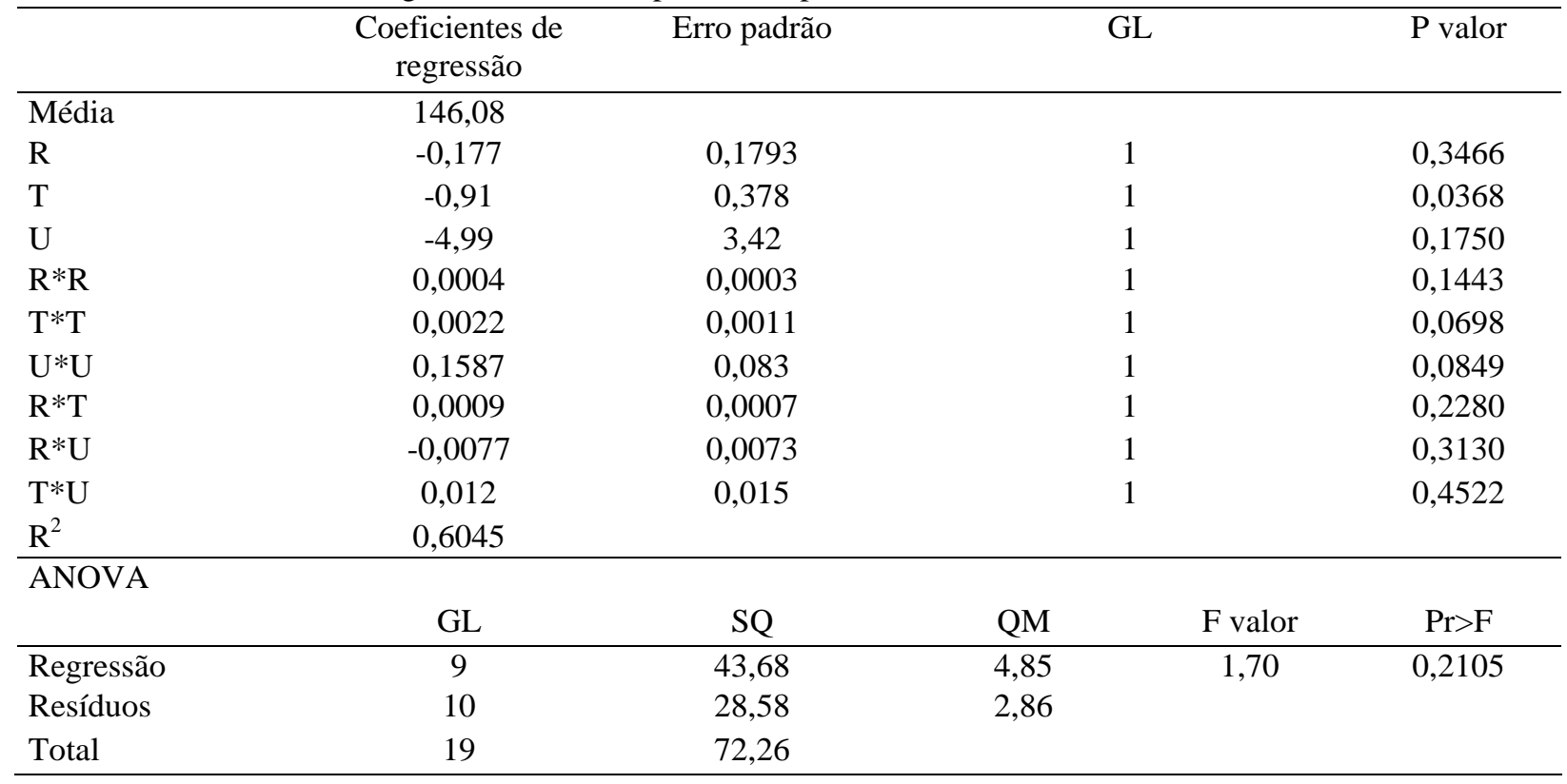

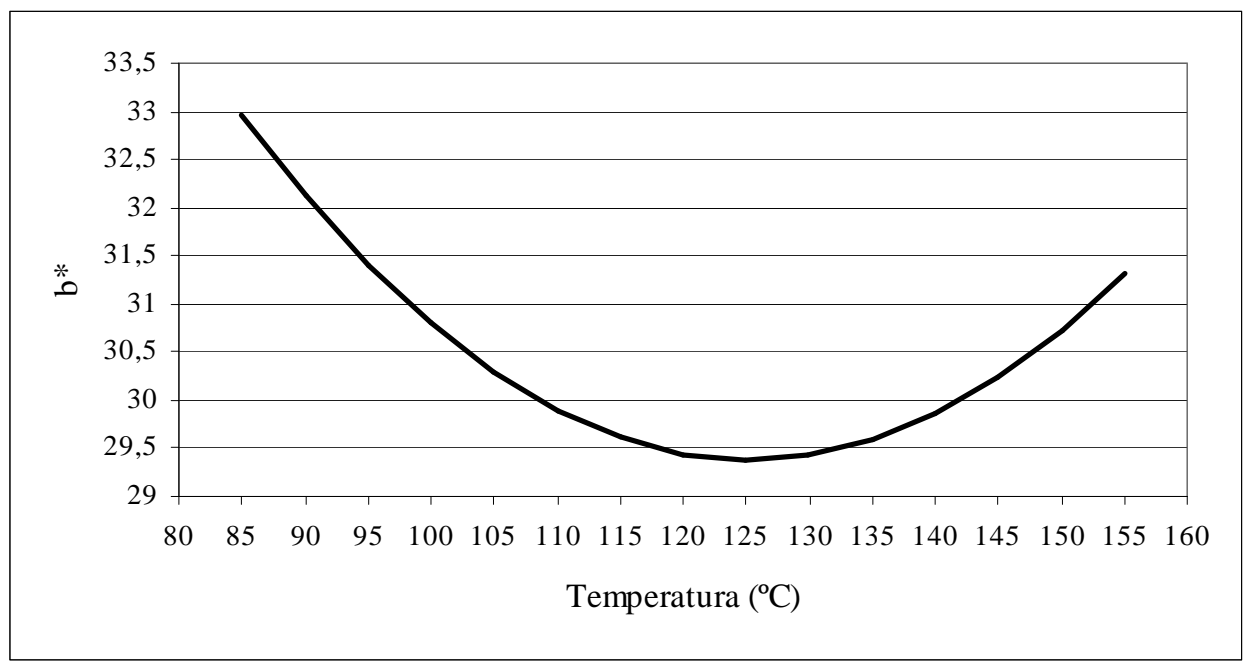

Figura 3 - Efeito da temperatura sobre o componente $b^{*}$ das farinhas extrusadas, sob condições de umidade da matéria-prima de $15 \%$ e rotação da rosca de 204 rpm.

Ainsworth et al. (2007), estudando o efeito da rotação da rosca e da adição do resíduo fibroso da fabricação de cerveja nas características físicas e nutricionais de snacks, sabor cebola, produzidos a partir de misturas de farinha de trigo, amido de milho, farinha de aveia e grão-de-bico, observaram variações nos componentes de cor (Hunter) com L variando de 51,7 a 65,3 , croma a de $-6,5$ a $-11,8$ e croma b variando de 12,8 a 20,8. As condições de operação foram: extrusor dupla rosca, rotação variando de 100 a $300 \mathrm{rpm}$, taxa de alimentação de $42 \mathrm{~kg} / \mathrm{h}$, temperatura na $2^{\mathrm{a}}$ zona de $80^{\circ} \mathrm{C}$ e na terceira zona de $100{ }^{\circ} \mathrm{C}$, umidade de $11,8 \%$. 


\section{Propriedades de pasta}

A viscosidade é uma das propriedades mais importantes dos materiais amiláceos. A curva de viscosidade representa o seu comportamento durante o aquecimento e permite avaliar as características da pasta formada por causa das modificações estruturais da molécula de amido e, também, a tendência à retrogradação, durante o resfriamento. Essas características determinam as propriedades funcionais das matérias-primas amiláceas e suas diversas aplicações industriais.

A viscosidade inicial (VI) a $25^{\circ} \mathrm{C}$, também chamada de viscosidade de pasta a frio, indica a capacidade das farinhas em absorver água a temperatura ambiente e formar uma pasta, gel ou líquido viscoso. A VI da farinha de mandioquinha-salsa crua foi de $5 \mathrm{RVU}$ e a VI das farinhas extrusadas variou de 14 a 54 RVU, mostrando o aumento dessa propriedade com a extrusão. Nas amostras extrusadas, o amido está gelatinizado, e torna possível a rápida hidratação dos grânulos a temperatura ambiente, sendo importante em produtos em que se deseja maior viscosidade a frio.

A análise de regressão evidenciou a influência significativa da umidade sobre a viscosidade inicial das farinhas extrusadas, obtendo-se um coeficiente de determinação de 71,01\% (Tabela 5).
Com o aumento da umidade ocorreu um aumento significativo da VI, mantendo-se a temperatura e a rotação nos pontos centrais $\left(120^{\circ} \mathrm{C}\right.$ e $\left.204 \mathrm{rpm}\right)$ (Figura 4). Os valores de viscosidade inicial se elevam com a prévia gelatinização da amostra e decrescem em condições severas de processamento em razão da despolimerização do amido com a cocção.

De acordo com Sebio (1996), o efeito da umidade na viscosidade inicial pode ser explicado pela lubrificação que o alto teor de água provoca na rosca do extrusor evitando, assim, maior ocorrência de degradação mecânica dos grânulos de amido, com conseqüente aumento de VI.

O pico de viscosidade (PV) ocorre após o início do aquecimento e antes do início do resfriamento da suspensão, no viscoamilógrafo. Se os tratamentos não forem tão severos, parte dos grânulos de amido conservam sua estrutura, apresentando um pico no perfil amilográfico (GUHA et al., 1998). Entretanto, no processo de extrusão, condições severas podem destruir a estrutura cristalina dos grânulos de amido resultando em ausência de pico.

A farinha de mandioquinha-salsa crua apresentou pico de viscosidade de $148 \mathrm{RVU}$, valor inferior ao relatado por Vieira (2004) para amido de mandioquinha-salsa (353 RVU), o que pode ser explicado pela presença de outros componentes na farinha.

Tabela 5 - Coeficientes de regressão e ANOVA, para a viscosidade inicial das farinhas extrusadas.

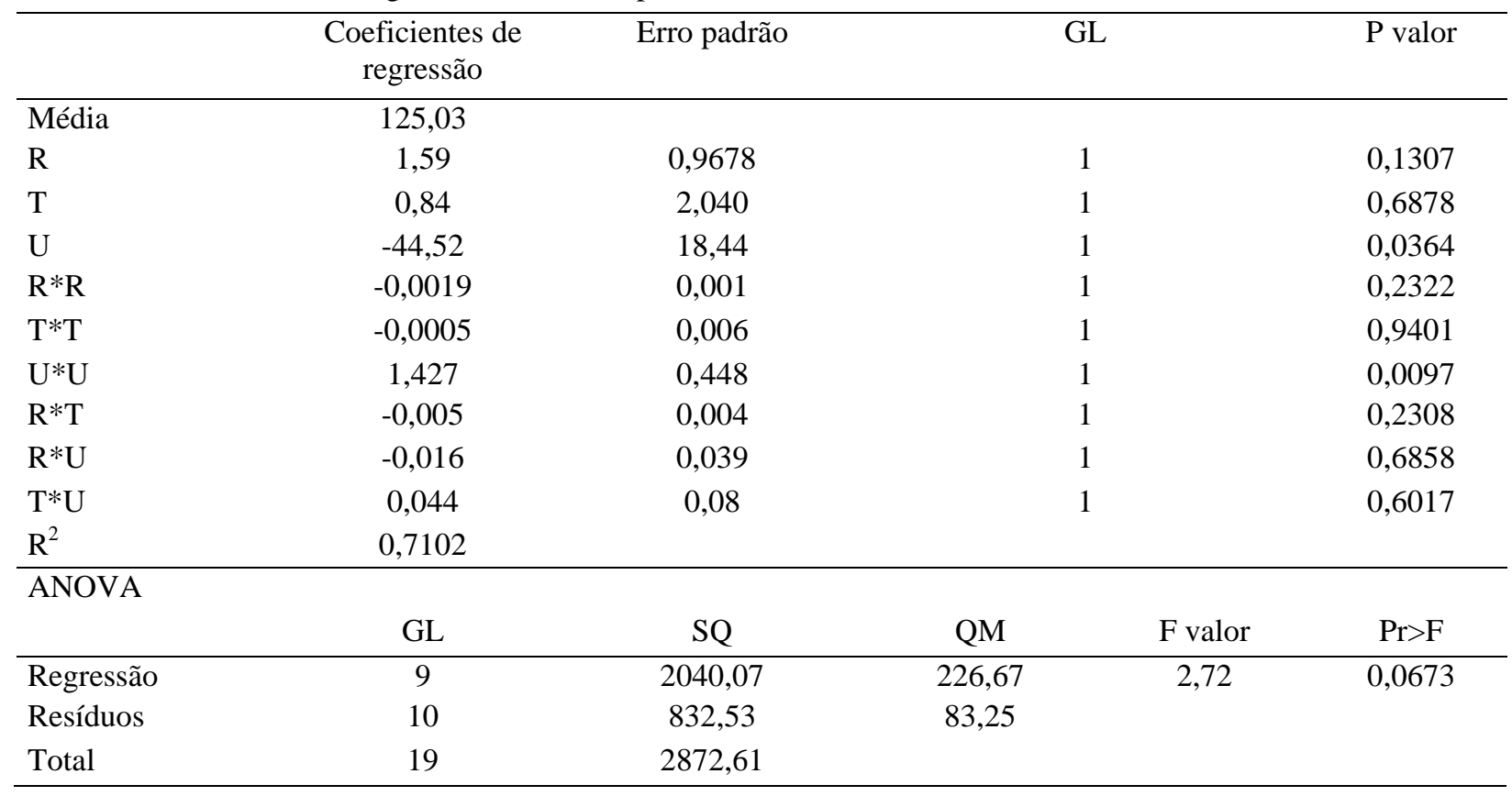


Os picos de viscosidade das farinhas extrusadas variaram de 8 a 56 RVU. Essa variação indica diferentes graus de gelatinização e destruição da estrutura cristalina dos grânulos entre os tratamentos efetuados.

A análise dos coeficientes de regressão mostrou não ter ocorrido efeito significativo da temperatura de extrusão e da rotação da rosca sobre o pico de viscosidade.
Entretanto, a umidade teve efeito sobre o pico de viscosidade das amostras extrusadas. $\mathrm{O}$ coeficiente de determinação $\left(\mathrm{R}^{2}\right)$ foi de $61,1 \%$ (Tabela 6 ).

Conforme observa-se na Figura 5, a condição intermediária de temperatura, rotação da rosca e umidade foi a que promoveu menor pico de viscosidade. Com o aumento da umidade ocorreu uma tendência a maiores picos, possivelmente pelo menor atrito.

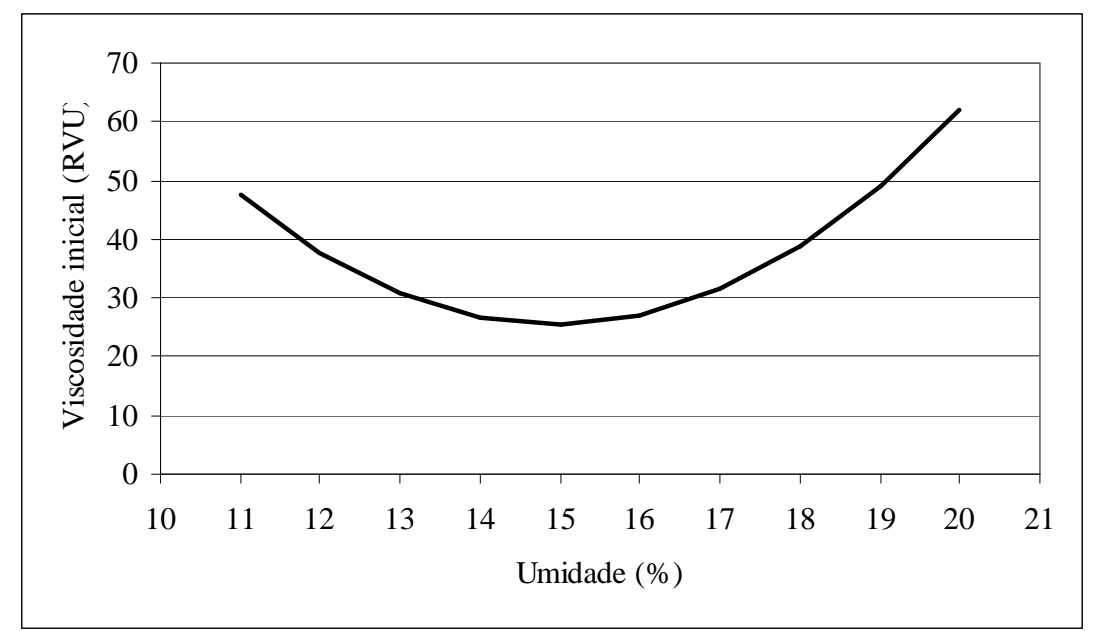

Figura 4 - Efeito do teor de umidade da matéria-prima sobre a viscosidade inicial (VI) das farinhas de mandioquinhasalsa extrusadas, sob condições de temperatura de $120^{\circ} \mathrm{C}$ e rotação da rosca de $204 \mathrm{rpm}$.

Tabela 6 - Coeficientes de regressão e ANOVA, para o pico de viscosidade das farinhas extrusadas.

\begin{tabular}{|c|c|c|c|c|c|}
\hline & $\begin{array}{l}\text { Coeficientes de } \\
\text { regressão }\end{array}$ & Erro padrão & & & $\mathrm{P}$ valor \\
\hline Média & $-33,24$ & & & & \\
\hline $\mathrm{R}$ & 1,82 & 1,15 & & & 0,1457 \\
\hline $\mathrm{T}$ & 2,67 & 2,44 & & & 0,2984 \\
\hline $\mathrm{U}$ & $-39,25$ & 22,01 & & & 0,1048 \\
\hline $\mathrm{R} * \mathrm{R}$ & $-0,002$ & 0,002 & & & 0,3791 \\
\hline $\mathrm{T} * \mathrm{~T}$ & $-0,005$ & 0,007 & & & 0,5272 \\
\hline $\mathrm{U}^{*} \mathrm{U}$ & 1,27 & 0,53 & & & 0,0383 \\
\hline $\mathrm{R} * \mathrm{~T}$ & $-0,008$ & 0,005 & & & 0,1229 \\
\hline $\mathrm{R} * \mathrm{U}$ & $-0,015$ & 0,05 & & & 0,7620 \\
\hline $\mathrm{T} * \mathrm{U}$ & 0,022 & 0,10 & & & 0,8231 \\
\hline $\mathrm{R}^{2}$ & 0,6107 & & & & \\
\hline \multicolumn{6}{|l|}{ ANOVA } \\
\hline & GL & SQ & QM & $\mathrm{F}$ valor & $\operatorname{Pr}>\mathrm{F}$ \\
\hline Regressão & 9 & 1680,44 & 206,72 & 1,74 & 0,1996 \\
\hline Resíduos & 10 & 1186,16 & 118,62 & & \\
\hline Total & 19 & 3046,60 & & & \\
\hline
\end{tabular}


Os maiores picos encontrados nas condições de baixa umidade $(<15 \%)$ estão relacionados aos valores de viscosidade inicial, pois as diferenças observadas entre estas variáveis foram mínimas nos tratamentos.

Whalen et al. (1997) avaliando as condições operacionais na extrusão de misturas de farinhas de arroz,

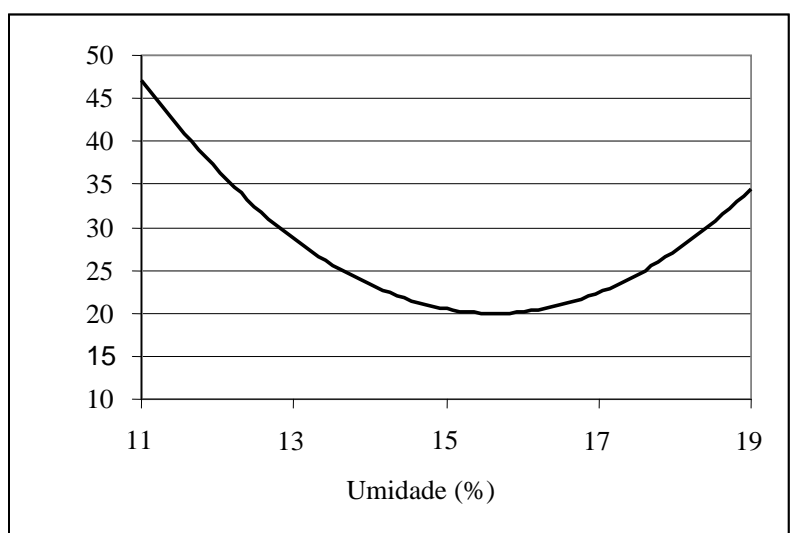

Figura 5 - Efeito do teor de umidade da matéria-prima sobre o pico de viscosidade (PV) das farinhas de mandioquinha-salsa extrusadas, sob condições de temperatura de $120^{\circ} \mathrm{C}$ e rotação da rosca de $204 \mathrm{rpm}$. trigo e milho, com a temperatura variando de 160 a $190{ }^{\circ} \mathrm{C}$ e a rotação mantida em $200 \mathrm{rpm}$, observaram que a diminuição da umidade de $29 \%$ para $15 \%$ resultou em redução significativa do pico de viscosidade das amostras.

A quebra da viscosidade $(\mathrm{QV})$ representa a diferença entre o pico de viscosidade e o menor valor de viscosidade após o pico, no perfil amilográfico. Através dessa propriedade é possível avaliar a estabilidade da pasta em altas temperaturas, sob agitação mecânica.

Os dados obtidos para quebra de viscosidade das farinhas extrusadas de mandioquinha-salsa variaram de 9,6 a 48 RVU. Esses valores foram inferiores aos obtidos para a farinha de mandioquinha-salsa antes da extrusão que foi de 96 RVU, o que era esperado, uma vez que os picos de viscosidade foram inferiores ao da farinha antes da extrusão. Da mesma foram que, para o pico de viscosidade, somente a umidade afetou significativamente a quebra de viscosidade das farinhas extrusadas (Tabela 7).

Nas condições intermediárias de temperatura de extrusão $\left(120^{\circ} \mathrm{C}\right)$, rotação da rosca (rpm) e umidade $(15 \%)$ observaram-se baixos valores de quebra de viscosidade (QV), nas farinhas extrusadas de mandioquinha-salsa (Figura 6).

Tabela 7 - Coeficientes de regressão e ANOVA para a quebra de viscosidade das farinhas extrusadas.

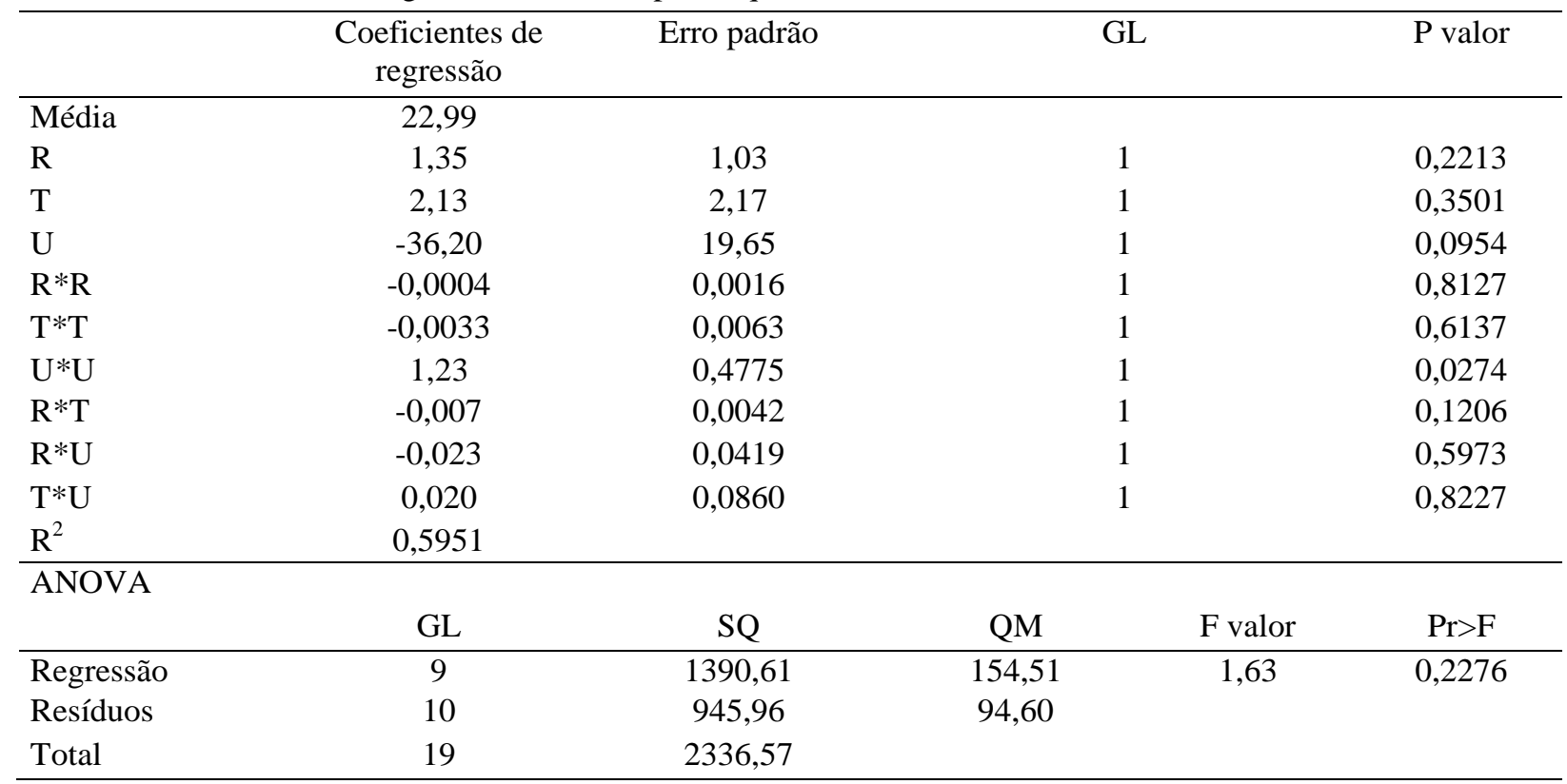




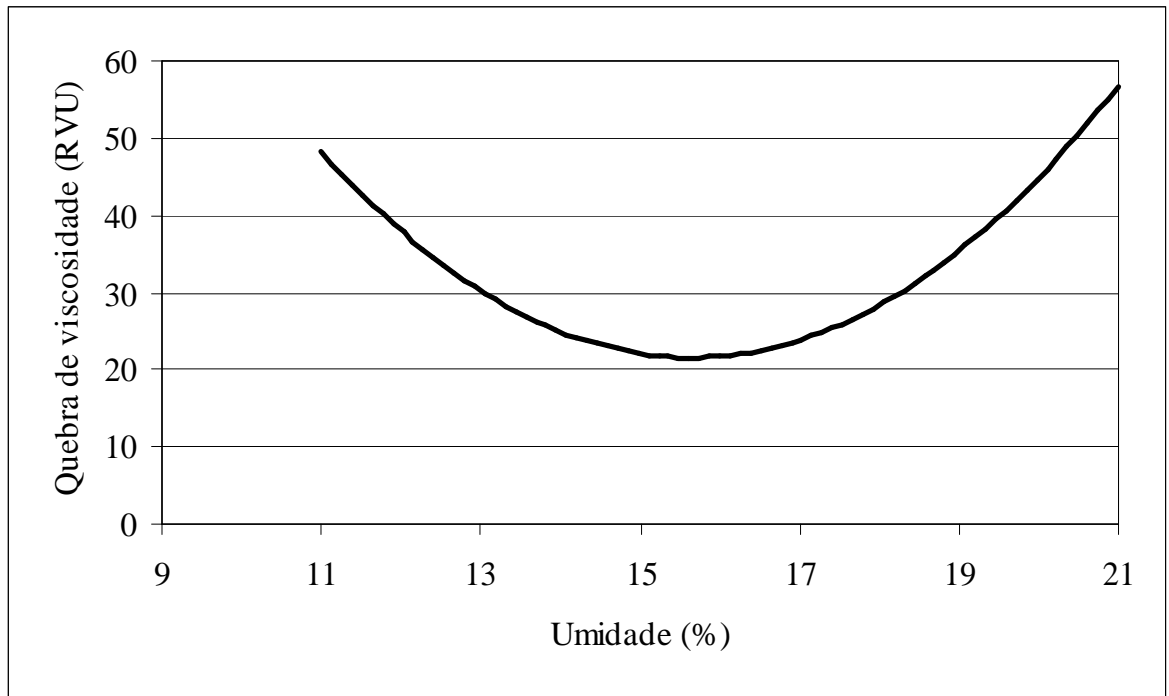

Figura 6 - Efeito do teor de umidade da matéria-prima sobre a quebra de viscosidade (QV) das farinhas de mandioquinhasalsa extrusadas, sob condições de temperatura de $120^{\circ} \mathrm{C}$ e rotação da rosca de $204 \mathrm{rpm}$.

Bhattacharya et al. (1999), estudando o efeito das condições de extrusão sobre as propriedades de pasta de misturas de farinha de batata e trigo, com a temperatura mantida em $160^{\circ} \mathrm{C}$, rotação variando de 200 a $400 \mathrm{rpm}$, observaram que quando a umidade subiu de $15,5 \%$ para $20,7 \%$ ocorreu um aumento significativo nos valores de $\mathrm{QV}$, concluindo que a elevada umidade poderia estar agindo como lubrificante, reduzindo a viscosidade de fusão.

A tendência à retrogradação (TR) mede a diferença entre a viscosidade final e o menor valor de viscosidade após o pico. Essa propriedade permite avaliar o comportamento da pasta após o seu resfriamento. $\mathrm{Na}$ farinha de mandioquinha-salsa, antes da extrusão, observou-se 39 RVU de tendência à retrogradação. Vieira (2004) analisando as propriedades de pasta do amido de mandioquinha-salsa observou baixa TR (37 RVU).

Os valores de tendência à retrogradação das farinhas de mandioquinha-salsa extrusadas variaram de 2 a 16 RVU. Esses valores baixos de TR indicam a severidade dos tratamentos, com a degradação dos polímeros, rompimento das estruturas moleculares e redução da capacidade de recristalização dos mesmos.

O modelo de regressão adotado para a variável TR foi significativo $(\mathrm{p}<0,05)$. Foram observados efeitos significativos dos três fatores sobre essa propriedade de pasta. O coeficiente de determinação foi de $81,6 \%$, indicando bom ajuste do modelo aos dados (Tabela 8).
Nas condições de rotação intermediária e temperatura mais elevada observaram-se os maiores valores de TR, entretanto, as condições extremas de rotação e temperatura, com a umidade no ponto central, ocasionaram baixa tendência à retrogradação nas amostras (Figura 7). Borba (2005) verificou TR variando de 26 a 29 RVU em farinhas de batata-doce extrusadas em rotação de rosca de $180 \mathrm{rpm}$ e valores de TR de 17 a 18 RVU em rotações elevadas (240 e $260 \mathrm{rpm}$ ).

A viscosidade final (VF) é uma característica que, em produtos extrusados, vai depender das modificações que ocorrem nas estruturas do grânulo de amido e das moléculas, durante o processamento.

Neste experimento, as viscosidades finais das farinhas de mandioquinha-salsa variaram de 4 a 26 RVU, valores inferiores ao observado na farinha antes da extrusão (91,58 RVU). Resultados semelhantes foram obtidos por Bhattacharya et al. (1999) que observaram viscosidade final de 106 RVU, em misturas de farinha de trigo e batata antes da extrusão e após a extrusão a VF variou de 11 a 18 RVU, nas diferentes condições empregadas.

O modelo de regressão adotado para a variável VF foi significativo $(\mathrm{p}<0,05)$. Foi observado efeito significativo da rotação e da temperatura. A umidade não apresentou efeito significativo sobre essa propriedade de pasta. O coeficiente de determinação foi de $80,6 \%$ (Tabela 9). 
Tabela 8 - Coeficientes de regressão e ANOVA para a tendência a retrogradação das farinhas extrusadas.

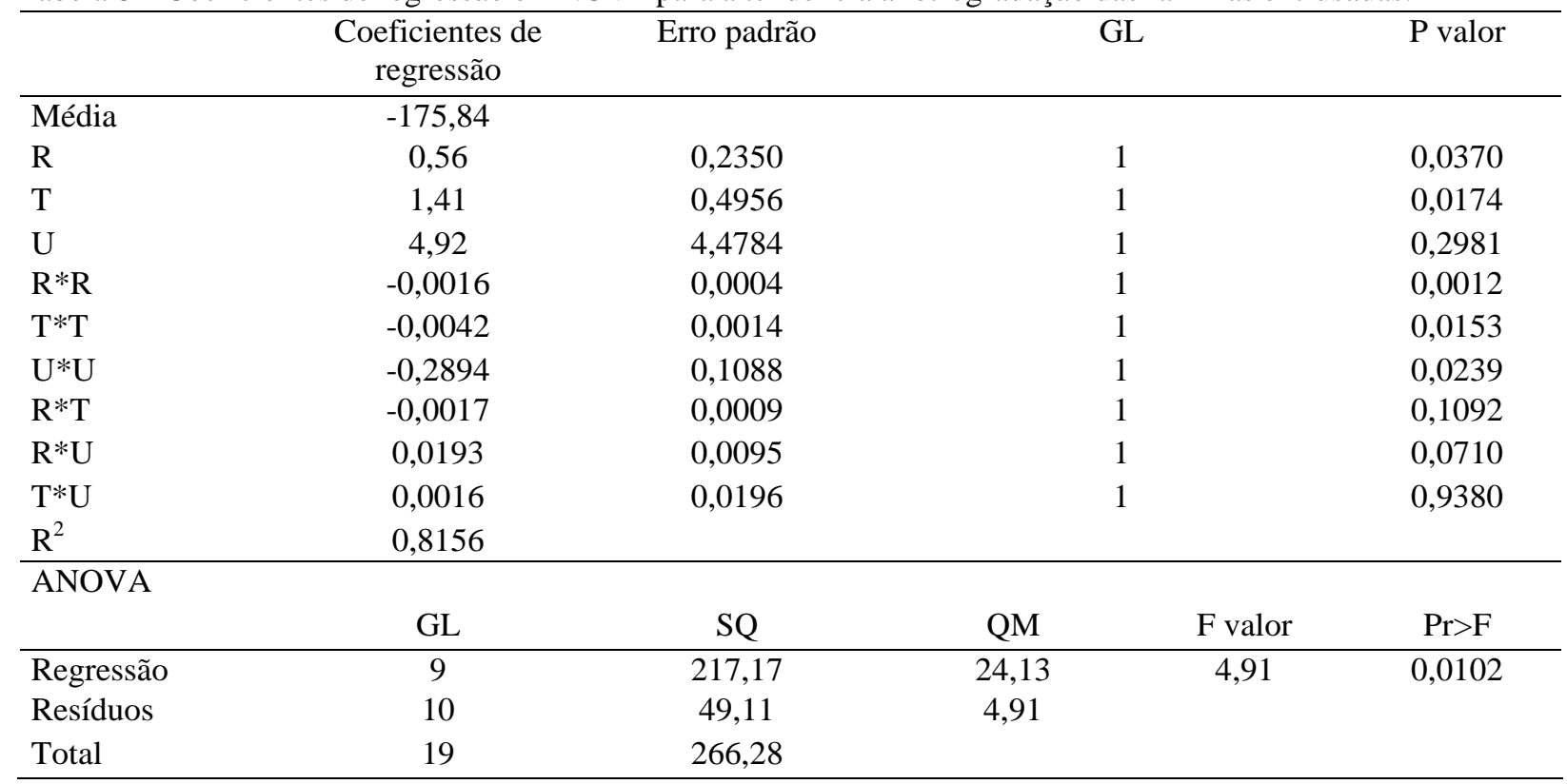

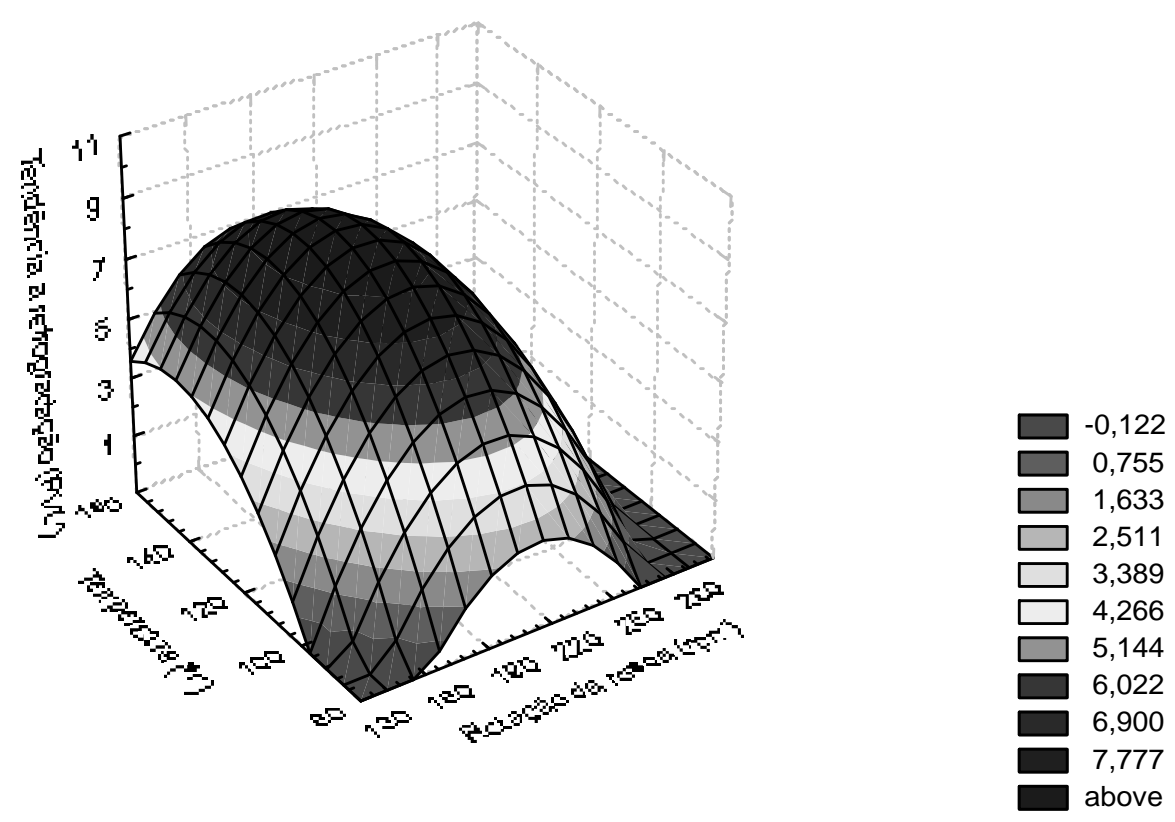

Figura 7 - Efeito da rotação da rosca e da umidade na tendência à retrogradação (TR) das farinhas extrusadas de mandioquinha-salsa, com temperatura de extrusão em $120^{\circ} \mathrm{C}$.

Nas condições extremas de temperatura e causando perda da capacidade de recristalização rotação a viscosidade final foi baixa, indicando e diminuindo a viscosidade no resfriamento rompimento das estruturas moleculares do amido, (Figura 8). 
Tabela 9 - Coeficientes de regressão e ANOVA, para a viscosidade final das farinhas extrusadas.

\begin{tabular}{|c|c|c|c|c|c|}
\hline & $\begin{array}{l}\text { Coeficientes de } \\
\text { regressão }\end{array}$ & Erro padrão & GL & & $\mathrm{P}$ valor \\
\hline Média & $-232,17$ & & & & \\
\hline $\mathrm{R}$ & 1,04 & 0,4025 & 1 & & 0,0270 \\
\hline $\mathrm{T}$ & 1,95 & 0,8487 & 1 & & 0,0445 \\
\hline $\mathrm{U}$ & 1,87 & 7,6695 & 1 & & 0,8121 \\
\hline $\mathrm{R} * \mathrm{R}$ & $-0,003$ & 0,0006 & 1 & & 0,0010 \\
\hline $\mathrm{T}^{*} \mathrm{~T}$ & $-0,006$ & 0,0025 & 1 & & 0,0484 \\
\hline $\mathrm{U}^{*} \mathrm{U}$ & $-0,25$ & 0,1863 & 1 & & 0,2151 \\
\hline $\mathrm{R} * \mathrm{~T}$ & $-0,0025$ & 0,0016 & 1 & & 0,1608 \\
\hline $\mathrm{R} * \mathrm{U}$ & 0,028 & 0,0164 & 1 & & 0,1230 \\
\hline $\mathrm{T}^{*} \mathrm{U}$ & 0,0039 & 0,033 & 1 & & 0,9103 \\
\hline $\mathrm{R}^{2}$ & 0,8060 & & & & \\
\hline \multicolumn{6}{|l|}{ ANOVA } \\
\hline & GL & SQ & $\mathrm{QM}$ & F valor & $\operatorname{Pr}>F$ \\
\hline Regressão & 9 & 598,39 & 66,49 & 4,62 & 0,0127 \\
\hline Resíduos & 10 & 144,03 & 14,40 & & \\
\hline Total & 19 & 742,42 & & & \\
\hline
\end{tabular}

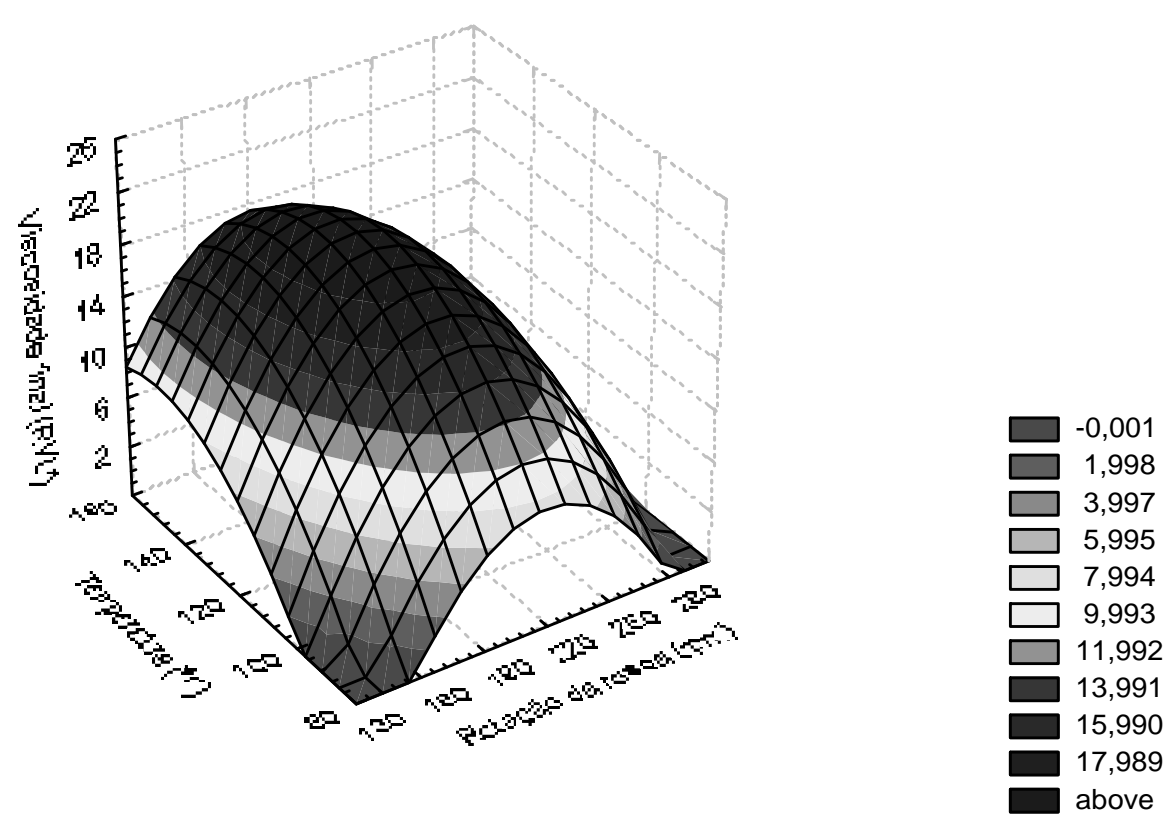

Figura 8 - Efeito da rotação da rosca e temperatura de extrusão na viscosidade final (VF) das farinhas extrusadas de mandioquinha-salsa, com umidade de $15 \%$ na matéria-prima.

\section{CONCLUSÃO}

As variações nas condições operacionais do processo de extrusão da farinha de mandioquinha-salsa promoveram importantes alterações nos componentes de cor e propriedades de pasta com interferência da umidade da farinha na luminosidade, croma $a^{*}$, nas viscosidades 
inicial e final, pico e quebra de viscosidade. A temperatura de extrusão e a rotação da rosca tiveram efeito na tendência à retrogradação e viscosidade final das farinhas extrusadas.

Considerando um possível uso da farinha extrusada de mandioquinha-salsa como produto instantâneo as condições de $17 \%$ de umidade, $120^{\circ} \mathrm{C}$ de temperatura de extrusão e $204 \mathrm{rpm}$ de rotação da rosca produziriam produtos com características adequadas para esse uso.

\section{REFERÊNCIAS BIBLIOGRÁFICAS}

AINSWORTH, P.; IBANOGLU, S.; PLUNKETT, A.; IBANOGLU, E.; STOJCESKA, V. Effect of brewers spent grain addtion and screw speed on the select physical and nutritional properties of an extruded snack. Journal of Food Engineering, Essex, v. 81, p. 702-709, 2007.

BADRIE, N.; MELLOWES, W. A. Effect of extrusion variables on cassava extrudates. Journal of Food Science, Chicago, v. 56, p. 1334-1337, 1991.

BHATTACHARYA, S.; SUDHA, M. L.; RABIN, A. Pasting characteristics of an extruded blend of potato and wheat. Journal of Food Engineering, Essex, v. 40, p. 107-111, 1999.

BORBA, A. L. Efeito de alguns parâmetros operacionais nas características físicas, físico-químicas e funcionais de extrusados da farinha de batata-doce(Ipomoea batatas). 2005. 98 f. Dissertação (Mestrado) - Escola Superior de Agricultura “Luiz de Queiroz”, Universidade de São Paulo, Piracicaba, 2005.

BORBA, A. M.; SARMENTO, S. B. S.; LEONEL, M. Efeito dos parâmetros de extrusão sobre as propriedades funcionais de extrusados da farinha de batata-doce. Ciência e Tecnologia de Alimentos, Campinas, v. 25, n. 4, p. 835843, 2005.

CÂMARA, F. L. A.; SANTOS, F. F. S. Cultura da araruta. In: CEREDA, M. P. (Coord.). Agricultura: tuberosas amiláceas latino americanas. São Paulo: Fundação Cargill, 2002. p. 519-532.

CHANG, Y. K.; HASHIMOTO, J. M.; ACIOLI-MOURA, R.; MARTINÉZ-FLORES, H. E.; MARTINÉZ-BUSTOS, F. Influence of extrusion condition on cassava starch and soybean protein concentrate blends. Acta Alimentaria,
Amsterdam, v. 30, n. 2, p. 189-203, 2001

COCHRAN, W. G.; COX, G. M. Experimental designs. 2. ed. New York: J. Wiley \& Sons, 1957. 611 p.

GUHA, M.; ALI, S. Z.; BHATTACHARYA, S. Effect of barrel temperature and screw speed on rapid viscoanylyzer pasting behavior of rice extruded. International Journal of Food Science and Technology, Oxford, v. 33, p. 259-266, 1998.

GUTKOSKI, L. C.; EL-DASH, A. A. Effect of extrusion process variables on physical and chemical properties of extruded oat products. Plant Foods for Human Nutricion, The Hague, v. 54, p. 315-325, 1999.

NEWPORT SCIENTIFIC. Operation manual series 4: instructions manual. Warriedwood, 1998. 123 p.

PEREIRA, A. S. Valor nutritivo da mandioquinha-salsa. Informe Agropecuário, Belo Horizonte, v. 19, n. 190, p. 1112, 1997.

SEBIO, L. Efeito de alguns parâmetros operacionais de extrusão nas propriedades físico-químicas da farinha de inhame (Dioscorea rotundata). 1996. Dissertação (Mestrado) - Universidade Estadual de Campinas, Campinas, 1996.

THAKUR, S.; SAXENA, D. C. Formulation of extruded snack food (gum based cereal-pulse blend): optimization of ingredients levels using response surface methodology. Lebensmittel Wissenschaft und Technologie, London, v. 33 , p. 354-361, 2000.

VIEIRA, F. C. Efeito do tratamento com calor e baixa umidade sobre características físicas e funcionais dos amidos de mandioquinha-salsa (Arracacia xanthorrhiza), batata-doce (Ipomoea batatas) e de gengibre (Zingiber officinale). 2004. 103 f. Dissertação (Mestrado) - Escola Superior de Agricultura “Luiz de Queiroz”, Piracicaba, 2004.

WHALEN, P. J.; BASON, M. L.; BOOTH, R. I.; WALKER, C. E.; WILLIAMS, P. J. Measurement of extrusion effects by viscosity profile using rapid viscoanalyser. Cereal Foods World, Minneapolis, v. 42, n. 6, p. 469-475, 1997. 